\title{
The Vadasz-Olek Model Regarded as a System of Coupled Oscillators
}

\author{
Eugen Magyari
}

Received: 13 February 2010 / Accepted: 12 March 2010 / Published online: 31 March 2010

(C) Springer Science+Business Media B.V. 2010

\begin{abstract}
The Vadasz-Olek model of chaotic convection in a porous layer is revisited in this article. The first-order differential equations of this Lorenz-type model are transformed in the governing equations of a damped nonlinear oscillator, modulated by a linear degenerated overdamped oscillator (relaxator) which in turn is coupled to former one by a nonlinear cross force. The benefit of this mechanical analogy is an intuitive picture of the regular and chaotic dynamics described by the Vadasz-Olek model. Thus, there turns out that the "eyes" of the chaotic attractor correspond to the minima of the potential energy of the modulated nonlinear oscillator having a double-well shape. Several new aspects of the subcritical and supercritical dynamic regimes are discussed in some detail.
\end{abstract}

Keywords Porous layer - Chaotic convection - Strange attractors · Nonlinear oscillations · Damping $\cdot$ Modulation

\section{Introduction}

Before the discovery of the deterministic low-dimensional chaos by Lorenz (1963), the physics of the twentieth century was a predominantly linear science. Electrodynamics and Quantum Mechanics as the leading physical theories of this time were defined on linear vector and function spaces, being governed by linear (Maxwell and Schrödinger) equations. The representation of the solutions by Fourier series and orthogonal polynomials are essentially linear methods, being based on the principle of the linear superposition. Although in the hydrodynamics, the general relativity, and the quantum field theories the important role of nonlinearities has been recognized from the very beginning, their effects could be managed in most of the cases by perturbation methods only.

Subsequent to the famous Los Alamos numerical experiment of Enrico Fermi, John Pasta, Stanislaw Ulam, and Mary Tsingou in 1953 (see e.g., Dauxois 2008), and a decade later,

E. Magyari (ه)

Departement Physik, Universität Basel, Klingelbergstr. 82, 4056 Basel, Switzerland

e-mail: magyari@bluewin.ch 
the discovery of the low dimensional turbulence by Lorenz (1963), it has definitively been recognized that the nonlinearities are responsible for a series of new universal phenomena which can neither be understood as simple perturbations of the linearity, nor as superposition of linear effects. The Los Alamos numerical experiment and the article of Lorenz (1963) mark actually the birth of the modern nonlinear physics. Subsequently, chaotic behavior and universal routes to chaos as consequences of nonlinearity have been found in population dynamical models (Li and Yorke 1975; May 1976), in single mode lasers (Haken 1975), in one-dimensional iteration maps (Feigenbaum 1979), in chemical reaction kinetics (Rössler 1976, 1979), in one-dimensional forced oscillations (Holmes 1979), and many other systems (see e.g., Eckmann 1981; Schuster 1984; Stoop and Steeb 2006).

With respect to the flow in fluid-saturated porous media Vadasz and Olek (1998) have proved that in the centrifugally induced convection in a rotating porous layer, the transition from steady to chaotic (turbulent) convection leads to a system of equations which are equivalent to the governing equations of the Lorenz model. Subsequently, similar results were reported by Vadasz and Olek $(1999,2000)$ and Vadasz $(1999,2003,2010)$ also for the convection in a porous layer heated from below. These results of Vadasz and Olek emphasize once more the existence in dissipative dynamical systems of some and universal routes to chaos. The aim of this article is to further contribute to the investigation of the Vadasz-Olek model of chaotic convection in a porous layer, by mapping its basic equations on the governing equation of a damped nonlinear oscillator modulated by a linear degenerated overdamped oscillator (relaxator) which is coupled to the damped oscillator by a nonlinear cross force self-consistently. This point-mechanical analogy provides an intuitive insight into the details both of the regular and chaotic dynamics of the model.

\section{The Vadasz-Olek Model}

According to the Vadasz-Olek model, the transition from steady to chaotic convection regime is described both in the centrifugally induced convection in a rotating porous layer (Vadasz and Olek 1998), as well as in the free convection in a porous layer heated from below (Vadasz and Olek 1999) by the Lorenz-type equations.

$$
\begin{aligned}
\dot{X} & =\alpha(Y-X), \\
\dot{Y} & =R X-Y-(R-1) X Z, \\
\dot{Z} & =4 \gamma(X Y-Z)
\end{aligned}
$$

subject to some initial conditions

$$
X(0)=X_{0}, \quad Y(0)=Y_{0}, \quad Z(0)=Z_{0},
$$

In the above equations, the variable $X$ is related to the stream function amplitude and the variables $Y$ and $Z$ to the temperature amplitudes. The dot denotes differentiation with respect to the dimensionless time $t, R$ stands for the scaled Rayleigh number, the parameter $\alpha$ is related to the Darcy-Prandtl number, and the parameter $\gamma$ to the front aspect ratio of the porous layer. The Vadasz-Olek model is isomorphic to the Lorenz model only in the range $R>1$. 


\section{Reduction to a System of Oscillators}

\subsection{Basic Equations}

It is known that an ordinary differential equation of order $n$ always can be decomposed in a system of equations of lower orders $n_{1}, n_{2}, \ldots$, so that $n_{1}+n_{2}+\cdots=n$, in particular in a system of $n$ equations of the first order. The converse is also possible. A system of first-order differential equations can be "compacted" into a system of different higher-order equations. Subsequent variable transformations also can be useful to further change the form of the resulting equations. Our aim in this Section is to reduce the system of three first-order equations (1) of the Vadasz-Olek model to the governing equations of an equivalent mechanical system which consists of two coupled oscillators. The benefit of this point-mechanical analogy is an intuitive picture of the regular and chaotic regimes of the model. To this end, we first express the variable $Y$ from Eq. 1a,

$$
Y=\frac{1}{\alpha}(\dot{X}+\alpha X)
$$

and substitute this in Eqs. 1b and 1c, which thus become

$$
\begin{gathered}
\ddot{X}+(1+\alpha) \dot{X}+\alpha(1-R) X=\alpha(1-R) X Z \\
\dot{Z}+4 \gamma Z=\frac{4 \gamma}{\alpha} X(\dot{X}+\alpha X)
\end{gathered}
$$

On the left-hand side of Eq. 4, we recognize the equation of a linear oscillator of spring constant $\alpha(1-R)$ in the presence of a linear damping force $-(1+\alpha) \dot{X}$. The left-hand side of Eq. 5 describes a degenerated linear oscillator, i.e., a spring-mass system of negligible mass (and spring constant $4 \gamma$ ), called also a relaxator. A relaxator can be imagined as e.g., a pingpong ball fixed to an expanded or compressed spring and immersed in honey. Being released, the ball approaches its equilibrium position $Z=0$ smoothly, according to the exponential law $\exp (-4 \gamma t)$. Other physical phenomena characterized by a similar relaxation dynamics are the switching-on and switching-off transients of various mechanical and electrical systems, and the charging and discharging processes of an electrical condenser in an LC-circuit. The oscillator (5) is coupled to oscillator (4) by a nonlinear force which depends both on the amplitude $X$ and the velocity $\dot{X}$ of the latter. The effect of the oscillator (5) on (4), on the other hand, can be imagined as a modulation of the spring constant $\alpha(1-R)$ of (4) by the elongation $Z$ of (5) in the sense that the linear force term $-[\alpha(1-R)] X$ is replaced by the modulated restoring force $-[\alpha(1-R)(1-Z)] X$.

Obviously, the above physical interpretation of the coupled oscillators (4), (5) is not unique. One could, e.g., replace the variable $Z$ in Eq. 4 by its expression obtained from Eq. 5 in terms of $X$ and $\dot{Z}$. Thus, one would obtain from (4) the equation of a nonlinear oscillator with a cubic nonlinearity and a nonlinear damping force which is similar to that of a van der Pol oscillator (for the latter, see e.g., Kneubühl 1997, p. 103). This nonlinearly damped nonlinear oscillator would then be coupled to the overdamped one (5) by a cross term proportional to $X \dot{Z}$. In addition to these operations, as mentioned above, we have the freedom to further change the form of Eqs. 4 and 5 by variable transformations, with the aim e.g., to remove the derivative $\dot{X}$ from Eq. 5. This latter task can be accomplished e.g. by the transformations

$$
X=\frac{\xi}{\sqrt{2 \gamma}}, \quad Z=\frac{1}{\alpha}\left(\xi^{2}+\eta\right)
$$


which have been used by Shimizu and Morioka (1978) in the investigation of the limit cycles occurring in the Lorenz model. In this way, Eqs. 4 and 5 go over in

$$
\begin{gathered}
\ddot{\xi}+(1+\alpha) \dot{\xi}+\alpha(1-R) \xi+(R-1) \xi^{3}=(1-R) \xi \eta \\
\dot{\eta}+4 \gamma \eta=2(\alpha-2 \gamma) \xi^{2}
\end{gathered}
$$

and Eq. 3 of $Y$ in

$$
Y=\frac{\dot{\xi}+\alpha \xi}{\alpha \sqrt{2 \gamma}}
$$

Furthermore, the initial conditions (2) are transformed to $\left(\xi(0) \equiv \xi_{0}, \dot{\xi}(0) \equiv \dot{\xi}_{0}, \eta(0) \equiv \eta_{0}\right)$ where

$$
\xi_{0}=\sqrt{2 \gamma} X_{0}, \dot{\xi}_{0}=\alpha \sqrt{2 \gamma}\left(Y_{0}-X_{0}\right), \quad \eta_{0}=\alpha Z_{0}-2 \gamma X_{0}^{2}
$$

The solution of the original initial value problem $(1, a, b, c),(2)$ is obtained in terms of the solution $(\xi, \eta)$ of the transformed initial value problem via Eqs. 6 and 9.

Now, on the left-hand side of Eq. 7, we recognize a slightly modified version of the linearly damped Duffing oscillator (see e.g., Kneubühl 1997, p. 98). The modification consists of the fact that the stiffness of the oscillator becomes negative for $R>1$, which implies that in this case, the linear part of the driving force does repel the particle away from the equilibrium state, instead to attract it. The left-hand side of Eq. 8 still describes a degenerated overdamped linear oscillator. The modified Duffing oscillator and the relaxator are coupled to each other by nonlinear forces which are proportional to $\xi \eta$ and $\xi^{2}$, respectively. Obviously, the cross coupling $\xi \eta$ can be seen also in this case as the amplitude modulation $-[(1-R)(\alpha-\eta)] \xi$ of the linear force term $-[\alpha(1-R)] \xi$. The nonlinear coupling and the amplitude modulation, along with the Duffing anharmonicity $\xi^{3}$, are responsible for the occurrence of a chaotic evolution in a certain parameter range of this linearly damped oscillator system associated with the Vadasz-Olek model $(1, a, b, c)$.

\subsection{The Stationary Points}

When $R \neq 1$, the basic equations (7) and (8) admit three constant solutions $\left(\xi_{i}, \eta_{i}\right), i=$ $1,2,3$ (called also stationary points, or fixed points) which are

$$
\left(\xi_{1}, \eta_{1}\right)=(0,0), \quad\left(\xi_{2,3}, \eta_{2,3}\right)=( \pm \sqrt{2 \gamma}, \alpha-2 \gamma) \quad(R \neq 1)
$$

For $R=1$, on the other hand, a continuous infinity of stationary points exist, namely

$$
\left(\xi_{*}, \eta_{*}\right)=\left(\xi_{*}, \frac{\alpha-2 \gamma}{2 \gamma} \xi_{*}^{2}\right) \quad(R=1)
$$

where $\xi_{*}$ is an arbitrary constant. When $R \rightarrow 1$, the stationary points (11) overlap in the special cases $\xi_{*}=0$ and $\xi_{*}= \pm \sqrt{2 \gamma}$ of Eq. 12 , respectively.

According to Eqs. 6 and 9 the stationary points (11) and (12) correspond in the original variables $(X, Y, Z)$ to

$$
\left(X_{i}, Y_{i}, Z_{i}\right)=\left(\frac{\xi_{i}}{\sqrt{2 \gamma}}, \frac{\xi_{i}}{\sqrt{2 \gamma}}, \frac{\xi_{i}^{2}+\eta_{i}}{\alpha}\right) \quad(R \neq 1)
$$


and

$$
\left(X_{*}, Y_{*}, Z_{*}\right)=\left(\frac{\xi_{*}}{\sqrt{2 \gamma}}, \frac{\xi_{*}}{\sqrt{2 \gamma}}, \frac{\xi_{*}^{2}}{2 \gamma}\right) \quad(R=1)
$$

\subsection{Limits of Stability}

The stability ranges of stationary points are determined by the eigenvalues $\lambda_{1,2,3}$ of the corresponding stability matrix $M$, i.e., by the roots of the characteristic polynomial $\operatorname{det}(M)$. The standard procedure to obtain the characteristic polynomial of the stationary points $\left(\xi_{j}, \eta_{j}\right), j=1,2,3, *$ given by Eqs. 11 and 12 is to linearize the governing equations (7) and (8) around the states $\left(\xi_{j}, \eta_{j}\right)$ by setting

$$
\xi=\xi_{j}+A e^{\lambda t}, \quad \eta=\eta_{j}+B e^{\lambda t}
$$

where $(A, B)$ and $B$ are small perturbations of $\left(\xi_{j}, \eta_{j}\right)$. It is easy to show that the matrix of the linearized system of Eqs. 7 and 8 (i.e., the "stability matrix" $M$ ) has the form

$$
M=\left(\begin{array}{cc}
\lambda^{2}+(\alpha+1) \lambda-(R-1)\left(\alpha-3 \xi_{j}^{2}-\eta_{j}\right) & (R-1) \xi_{j} \\
-4(\alpha-2 \gamma) \xi_{j} & \lambda+4 \gamma
\end{array}\right)
$$

Thus, the eigenvalue equation $\operatorname{det}(M)=0$ is

$$
(\lambda+4 \gamma)\left[\lambda^{2}+(\alpha+1) \lambda-(R-1)\left(\alpha-3 \xi_{j}^{2}-\eta_{j}\right)\right]+4(\alpha-2 \gamma)(R-1) \xi_{j}^{2}=0
$$

In case of the stationary point, $\left(\xi_{1}, \eta_{1}\right)=(0,0)$, which corresponds to the thermal conduction regime of the quiescent fluid (in thermal equilibrium with the porous matrix) Eq. 17 reduces to

$$
(\lambda+4 \gamma)[(\lambda+1)(\lambda+\alpha)-\alpha R]=0
$$

The corresponding eigenvalues are

$$
\lambda_{1}=-4 \gamma, \quad \lambda_{2,3}=-\frac{1+\alpha}{2}\left(1 \pm \sqrt{1-\frac{4 \alpha(1-R)}{(1+\alpha)^{2}}}\right)
$$

All three eigenvalues (19) are negative as long as $R<1$. Therefore, the stationary point $\left(\xi_{1}, \eta_{1}\right)=(0,0)$ is stable for $R<R_{c 1}$ and unstable for $R>R_{c 1}$ where $R_{c 1}=1$ is the first critical Rayleigh number of the Vadasz-Olek model where a pitchfork bifurcation occurs.

In case of the stationary points, $\left(\xi_{2,3}, \eta_{2,3}\right)=( \pm \sqrt{2 \gamma}, \alpha-2 \gamma)$, which correspond to the uniform convection states of the fluid, Eq. 17 reduces to

$$
\lambda^{3}+(4 \gamma+\alpha+1) \lambda^{2}+4 \gamma(\alpha+R) \lambda+8 \gamma \alpha(R-1)=0
$$

The eigenvalues $\lambda_{i}, i=1,2,3$ are related to the coefficients of Eq. 20 by the relationships

$$
\begin{aligned}
& \lambda_{1}+\lambda_{2}+\lambda_{3}=-(4 \gamma+\alpha+1), \\
& \lambda_{1} \lambda_{2}+\lambda_{1} \lambda_{3}+\lambda_{2} \lambda_{3}=4 \gamma(\alpha+R), \\
& \lambda_{1} \lambda_{2} \lambda_{3}=-8 \gamma \alpha(R-1)
\end{aligned}
$$

It is seen that the sum of the three eigenvalues is always negative and does not depend on $R$. Owing to the first and third Eq. 21, in the range $R<1$, one of the eigenvalues must always be positive. Accordingly, the stationary points $\left(\xi_{2,3}, \eta_{2,3}\right)$ are unstable for $R<1$. In the range $R>1$, on the other hand, on the same reason, at least one of the three eigenvalues, $\lambda_{1}$, say, 
must always be negative. Consequently, the stability of the stationary points $\left(\xi_{2,3}, \eta_{2,3}\right)$ in the range $R>1$ is decided by the behavior of the other two eigenvalues, $\lambda_{2}$ and $\lambda_{3}$. It is known from the previous study (Vadasz and Olek 1998), that there always exists a certain value $R=\bar{R}>1$ of the Rayleigh number so that (i) also the eigenvalues $\lambda_{2}$ and $\lambda_{3}$ are negative when $1<R \leq \bar{R}$; (ii) at $R=\bar{R}, \lambda_{2}$, and $\lambda_{3}$ become coincident, $\lambda_{2}=\lambda_{3} \equiv \bar{\lambda}<0$; (iii) in a certain interval $\bar{R}<R<R_{c 2}$ above of $\bar{R}$, the eigenvalues $\lambda_{2}$ and $\lambda_{3}$ become complex conjugate but their real part remains still negative, $\operatorname{Re}\left(\lambda_{2}\right)=\operatorname{Re}\left(\lambda_{3}\right)<0$; (iv) at $R=R_{c 2}$ the real part of the complex conjugate eigenvalues $\lambda_{2}$ and $\lambda_{3}$ vanishes, $\operatorname{Re}\left(\lambda_{2}\right)=$ $\operatorname{Re}\left(\lambda_{3}\right)=0$; and (v) for $R>R_{c 2}$ the real part of $\lambda_{2}$ and $\lambda_{3}$ becomes positive. These properties of the eigenvalues imply that $\bar{R}$ represents precisely that threshold value of $R$ where the stable simple nodes associated with the stationary points $\left(\xi_{2,3}, \eta_{2,3}\right)$ become stable spiral nodes. Furthermore, the upper bound $R=R_{c 2}$ of the interval $\bar{R}<R<R_{c 2}$ represents the second critical Rayleigh number of the Vadasz-Olek model above of which the stationary points $\left(\xi_{2,3}, \eta_{2,3}\right)$ become unstable. Accordingly, at $R=R_{c 2}$ a Hopf bifurcation occurs.

The value of the critical Rayleigh number $R_{c 2}$ can easily be calculated as follows. According to the above definition of $R_{c 2}$, at $R=R_{c 2}$, one has $\lambda_{2}=-\lambda_{3} \equiv i \lambda_{c}$. Thus, Eqs. 21 reduce at $R=R_{c 2}$ to

$$
\begin{aligned}
& \lambda_{1}=-(4 \gamma+\alpha+1), \\
& \lambda_{c}^{2}=4 \gamma\left(\alpha+R_{c 2}\right), \\
& \lambda_{1} \lambda_{c}^{2}=-8 \gamma \alpha\left(R_{c 2}-1\right)
\end{aligned}
$$

These equations yield for $R_{c 2}$ and the corresponding eigenvalues the expressions

$$
R_{c 2}=\frac{\alpha(3+\alpha+4 \gamma)}{\alpha-1-4 \gamma}, \lambda_{1}=-(4 \gamma+\alpha+1), \lambda_{2}=-\lambda_{3} \equiv i \lambda_{c}, \lambda_{c}=\sqrt{\frac{8 \gamma \alpha(1+\alpha)}{\alpha-1-4 \gamma}}
$$

Thus, at the critical Rayleigh number $R_{c 2}$, the stationary points $\left(\xi_{2,3}, \eta_{2,3}\right)$ possess a stable eigenmode which is associated with the eigenvalue $\lambda_{1}=-(4 \gamma+\alpha+1)$ and two neutrally stable eigenmodes associated with the imaginary eigenvalues $\lambda_{2}=-\lambda_{3} \equiv i \lambda_{c}$ given by Eqs. 23 . Therefore, the convection fixed points $\left(\xi_{2,3}, \eta_{2,3}\right)$ are stable in the range $1<R<R_{c 2}$ and become unstable for $R>R_{c 2}$. The critical value $R_{c 2}$ marks the transition to a chaotic evolution of the system. However, it is important to emphasize that $R_{c 2}$ is positive only for $\alpha>1+4 \gamma$. In this case, $R_{c 2}$ is also larger than 1 and goes to $+\infty$ when $\alpha$ approaches the value $1+4 \gamma$ from above. When, however, $\alpha<1+4 \gamma, R_{c 2}$ becomes negative, i.e., unphysical. This means that the stationary points $\left(\xi_{2,3}, \eta_{2,3}\right)$ are stable for all $R>1$ when $\alpha \leq 1+4 \gamma$ (see also Sects. 3.8 and 4.3). In other words, in the parameter range $\alpha \leq 1+4 \gamma$, no chaotic dynamics can arise (see also Sect. 4.3). Further properties of the characteristic values $\bar{R}$ and $R_{c 2}$ of the Rayleigh number will be discussed in Sects. 3.7 and 3.8 in more detail.

In case of the stationary points $\left(\xi_{*}, \eta_{*}\right)$ given by Eq. 12 and corresponding to $R=1$, Eq. 17 reduces to

$$
(\lambda+4 \gamma)(\lambda+\alpha+1) \lambda=0(R=1)
$$

Equation 24 which, in turn, coincides with the case $R=1$ of Eqs. 18 and 20, admits two negative and one vanishing eigenvalue $\lambda$. This means that the continuous infinity of stationary points corresponding to the first critical Rayleigh number $R=R_{c 1}=1$, are all marginally (neutrally) stable uniform solutions of the Vadasz-Olek model (see also Sect. 4.2). 


\subsection{The Special Case $\alpha=1$}

In this case, the three roots of the characteristic Eq. 20 can be given in the simple form

$$
\lambda_{1}=-2, \quad \lambda_{2,3}=-2 \gamma \pm 2 \sqrt{\gamma(1+\gamma-R)}
$$

It is readily seen that the threshold value $\bar{R}$ for which $\lambda_{2}$ and $\lambda_{3}$ become coincident, $\lambda_{2}=$ $\lambda_{3} \equiv \bar{\lambda}<0$, as well as the corresponding values of $\lambda_{1}$ and $\bar{\lambda}$ are

$$
\bar{R}=1+\gamma, \quad \lambda_{1}=-2, \quad \lambda_{2,3} \equiv \bar{\lambda}=-2 \gamma
$$

It is easy to check that Eqs. 26 actually give a solution of the system of Eqs. 21 for $\alpha=1$. This, however, is not the single solution for $\alpha=1$. There also exists a second solution for $\alpha=1$, namely

$$
\bar{R}=3-\frac{1}{\gamma}, \quad \lambda_{1}=2(1-2 \gamma), \quad \lambda_{2,3} \equiv \bar{\lambda}=-2
$$

This solution is physically meaningful only for $\gamma=1 / 2$ (see also Sect. 3.7).

\subsection{The Special Case $\alpha=2 \gamma$}

Similar to the case $\alpha=1$ discussed above, the case $\alpha=2 \gamma$ also belongs to the parameter range $\alpha \leq 1+4 \gamma$ in which the stationary points $\left(\xi_{2,3}, \eta_{2,3}\right)$ are stable for all $1<R<\infty$, i.e., where no chaotic evolution of the system can occur (see also Sect. 4.3). In this case, the roots of the characteristic polynomial are

$$
\lambda_{1}=-4 \gamma, \quad \lambda_{2,3}=-\frac{1}{2}\left(1+2 \gamma \pm \sqrt{(1+2 \gamma)^{2}-16 \gamma(R-1)}\right)
$$

It is seen that the threshold value $\bar{R}$ for which $\lambda_{2}$ and $\lambda_{3}$ become coincident, $\lambda_{2}=\lambda_{3} \equiv \bar{\lambda}<0$, and the corresponding values of $\lambda_{1}$ and $\bar{\lambda}$ are

$$
\bar{R}=\frac{4 \gamma^{2}+20 \gamma+1}{16 \gamma}, \quad \lambda_{1}=-4 \gamma, \quad \lambda_{2,3} \equiv \bar{\lambda}=-\frac{1+2 \gamma}{2}
$$

In the range $R>\bar{R}$, the eigenvalues $\lambda_{2,3}$ become complex conjugate, but their real part remains still negative. It is easy to show that $\bar{R} \geq 1.5$ for all $\gamma>0$, the minimum $\bar{R}_{\text {min }}=1.5$ being reached for $\gamma=0.5$.

Similar to the case $\alpha=1$, Eqs. 29 do not represent the single solution of the system of Eqs. 21 for $\alpha=2 \gamma$. Indeed, a second solution is

$$
\bar{R}=2(1-\gamma), \lambda_{1}=2 \gamma-1, \lambda_{2,3} \equiv \bar{\lambda}=-4 \gamma
$$

It is easily seen that, as it must be, Eqs. 29 and 30 taken for $\gamma=1 / 2$ coincide with Eqs. 26 and (27), respectively.

\subsection{Some Limiting Cases}

In some limiting cases, for the roots of the eigenvalue equation (20), simple approximate formulas can be found. Three such cases will be discussed below.

\footnotetext{
Case $\alpha \rightarrow 0, R>1$
} 
The threshold value $\bar{R}$ and the corresponding eigenvalues in this case can be approximated to the leading order by

$$
\bar{R}=\frac{(1+4 \gamma)^{2}}{16 \gamma}, \quad \lambda_{1}=-2\left(\frac{1-4 \gamma}{1+4 \gamma}\right)^{2} \alpha, \quad \lambda_{2,3} \equiv \bar{\lambda}=-\frac{1+4 \gamma}{2}
$$

Case $\alpha \rightarrow \infty$

In this case, $\bar{R}$ and the corresponding eigenvalues can be approximated by

$$
\bar{R}=1+\frac{\gamma}{2}, \quad \lambda_{1}=-\alpha, \quad \lambda_{2,3} \equiv \bar{\lambda}=-2 \gamma
$$

Furthermore, the critical Rayleigh number and the corresponding eigenvalues can be approximated for $\alpha \rightarrow \infty$ by

$$
R_{c 2}=\alpha, \quad \lambda_{1}=-\alpha, \quad \lambda_{2,3}= \pm 2 i \sqrt{2 \gamma \alpha}
$$

Case $R \rightarrow \infty$

In this case, the eigenvalues can be approximated by

$$
\lambda_{1}=-2 \alpha, \quad \lambda_{2,3} \rightarrow \frac{\alpha-(1+4 \gamma)}{2} \pm 2 i \sqrt{\gamma R}
$$

The real part of the above $\lambda_{2,3}$ is positive when $\alpha>1+4 \gamma$ and negative when $\alpha<1+4 \gamma$. In the latter case, as mentioned above, no chaotic evolution of the system is possible.

\subsection{The Threshold $\bar{R}$ to Spiral Nodes}

The threshold value $\bar{R}$ of the Rayleigh number is an important physical characteristic of the subcritical regime of the Vadasz-Olek model indicating the occurrence of a complex conjugate eigenvalue-pairs having negative real part when $\bar{R}<R<R_{c 2}$. Thus, the parameter range $\bar{R}<R<R_{c 2}$ is the "habitat" of the stable spiral nodes. Based on this reason, the dependence of $\bar{R}$ on the parameter $\alpha$ for a specified value of $\gamma$ is a basic feature of the under-critical dynamics. The aim of this Section is to discuss the behavior of the function $\bar{R}(\alpha)$ for $\gamma=0.5$ which is a typical value of $\gamma$ for the convection in porous media (Vadasz and Olek 1999).

According to the above definition of $\bar{R}$, at $R=\bar{R}$ the negative eigenvalues $\lambda_{2}$ and $\lambda_{3}$ become coincident, $\lambda_{2}=\lambda_{3} \equiv \bar{\lambda}<0$. Thus, Eqs. 21 reduce at $R=\bar{R}$ to

$$
\begin{aligned}
& \lambda_{1}+2 \bar{\lambda}=-(4 \gamma+\alpha+1), \\
& 2 \lambda_{1} \bar{\lambda}+\bar{\lambda}^{2}=4 \gamma(\alpha+\bar{R}), \\
& \lambda_{1} \bar{\lambda}^{2}=-8 \gamma \alpha(\bar{R}-1)
\end{aligned}
$$

We begin the discussion of the system of Eqs. 35 with the special situation in which not only $\lambda_{2}$ and $\lambda_{3}$, but all three negative eigenvalues $\lambda_{i}$ become coincident, and denote the corresponding values of $\bar{\lambda}, \bar{R}$, and $\alpha$ by $\bar{\lambda}_{*} \equiv \lambda_{1}=\lambda_{2}=\lambda_{3}, \bar{R}_{*}$, and $\alpha_{*}$, respectively. In this case, the eigenvalue equation (20) reduces to $\left(\lambda-\bar{\lambda}_{*}\right)^{3}=0$, and Eqs. 35 to

$$
\begin{aligned}
& 3 \bar{\lambda}_{*}=-\left(4 \gamma+\alpha_{*}+1\right), \\
& 3 \bar{\lambda}_{*}^{2}=4 \gamma\left(\alpha_{*}+\bar{R}_{*}\right), \\
& \bar{\lambda}_{*}^{3}=-8 \gamma \alpha_{*}\left(\bar{R}_{*}-1\right)
\end{aligned}
$$


This system of equations yields for $\bar{\lambda}_{*}$ and $\bar{R}_{*}$ the simple expressions

$$
\begin{aligned}
& \bar{\lambda}_{*}=-\frac{1}{3}\left(1+\alpha_{*}+4 \gamma\right), \\
& \bar{R}_{*}=1+\frac{1}{\gamma \alpha_{*}}\left(\frac{1+\alpha_{*}+4 \gamma}{6}\right)^{2}
\end{aligned}
$$

where $\alpha_{*}$ is the positive root of the cubic equation

$$
17 \alpha^{3}+3(11-28 \gamma) \alpha^{2}+3\left(5-32 \gamma+80 \gamma^{2}\right) \alpha-(1+4 \gamma)^{3}=0
$$

For $\gamma=0.5$, Eqs. 37 and 38 furnish the solution

$$
\begin{aligned}
& \alpha_{*}=\frac{3}{17}\left(1-2^{4 / 3}+2^{8 / 3}\right)=0.852311 \\
& \bar{\lambda}_{*}=-\frac{1}{17}\left(18-2^{4 / 3}+2^{8 / 3}\right)=1.284104 \\
& \bar{R}_{*}=\frac{3}{289}\left(129+7 \cdot 2^{4 / 3}+3 \cdot 2^{5 / 3}\right)=1.62107
\end{aligned}
$$

While the (physical) solution of the system of Eqs. 35 in the triple-root case (39) is unique, in the double root case $\lambda_{1}<\lambda_{2}=\lambda_{3} \equiv \bar{\lambda}<0$, non-unique physical solutions may exist. A specific example in this respect (for the already selected value $\gamma=0.5$ ) is the case $\alpha=0.9$ in which for the sets of values $\left(\bar{R}, \lambda_{1}, \bar{\lambda}\right)$, the following three solutions emerge:

$$
\begin{aligned}
& \left(\bar{R}, \lambda_{1}, \bar{\lambda}\right)_{1}=(1.01666,-0.015899,-1.94205), \\
& \left(\bar{R}, \lambda_{1}, \bar{\lambda}\right)_{2}=(1.47878,-0.654565,-1.62272), \quad(\alpha=0.9) \\
& \left(\bar{R}, \lambda_{1}, \bar{\lambda}\right)_{3}=(1.565812,-1.72954,-1.08523)
\end{aligned}
$$

For the sake of transparency, in Fig. 1 the solution $\bar{R}(\alpha)$ of the system of Eqs. 35 has been plotted in the range $0 \leq \alpha \leq 5$ of the parameter $\alpha$. The curve $\bar{R}(\alpha)$ consists of three branches which are highlighted by colors. The coordinates $(\alpha, \bar{R})$ of the points $A, B$, and $C$ marked by dots are indicated in Fig. 1 . The $\bar{R}$-coordinate 1.125 of the point $A$ results from the first Eq. 31 for $\gamma=0.5$. Point $B$ corresponds to the case (39) of the three coincident eigenvalues. The dashed line is the horizontal asymptote of the green-branch of the curve $\bar{R}(\alpha)$ and, according to the first Eq. 32 , corresponds to the value $\bar{R}(\infty)=1.25$. Figure 1 also shows that $\bar{R}$ is a one-valued function of $\alpha$ except for the interval $\alpha_{*} \leq \alpha \leq 1$, where its is two-valued at the ends $\alpha=\alpha_{*}$ and $\alpha=1$, and three-valued inside of this interval. The two values of $\bar{R}$ corresponding to $\alpha=\alpha_{*}$ are $\bar{R}=\bar{R}_{*}=1.62107$ (point $B$ ) and $\bar{R}=1.024252$ (projection of point $B$ on the red-branch). In the latter case, the eigenvalues are $\lambda_{1}=-0.022549$ and $\lambda_{2}=\lambda_{3} \equiv \bar{\lambda}=-1.91488$. The two values of $\bar{R}$ corresponding to $\alpha=1$ are $\bar{R}=1$ (point $C$ ) and $\bar{R}=1.5$ (projection of point $C$ on the green-branch). In the former case, the eigenvalues are $\lambda_{1}=0$ and $\lambda_{2}=\lambda_{3} \equiv \bar{\lambda}=-2$, and in the latter case, $\lambda_{1}=-2$ and $\lambda_{2}=\lambda_{3} \equiv \bar{\lambda}=-1$. All these results can be obtained from Eqs. 27 (point $C$ ) and Eqs. 26 (projection on the green-branch) for $\gamma=0.5$, respectively. We may summarize that Eqs. 35 admit a unique physical solution $\bar{R}$ for all $\alpha>0$, except for the interval $\alpha_{*} \leq \alpha \leq 1$ where two or three physical solutions arise. The existence domain of the spiral nodes associated with stationary points $\left(\xi_{2,3}, \eta_{2,3}\right)$ is bounded from below by the red and green curve of Fig. 1, except for the narrow domain between the blue and green curve in the interval $\alpha_{*} \leq \alpha \leq 1$, where simple nodes (i.e., three negative eigenvalues) occur. 


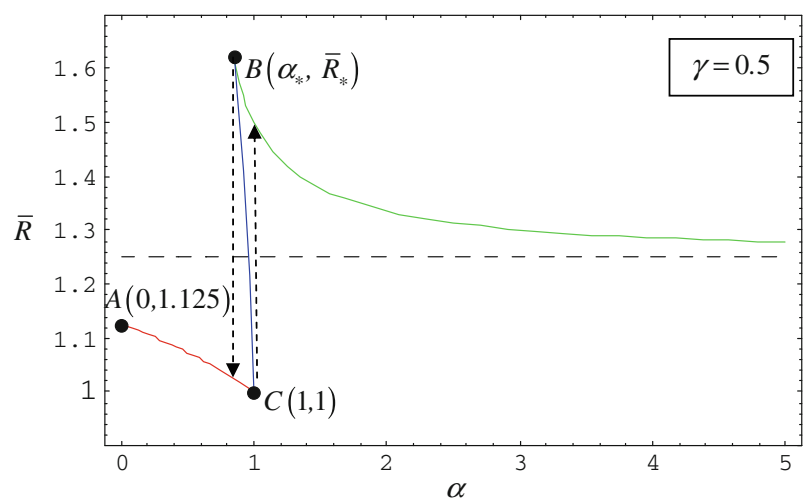

Fig. 1 The threshold value $\bar{R}$ is shown as a function of the parameter $\alpha$ for $\gamma=0.5$. The coordinates of the point $B$ are $\alpha_{*}=0.852311, \bar{R}_{*}=1.62107$

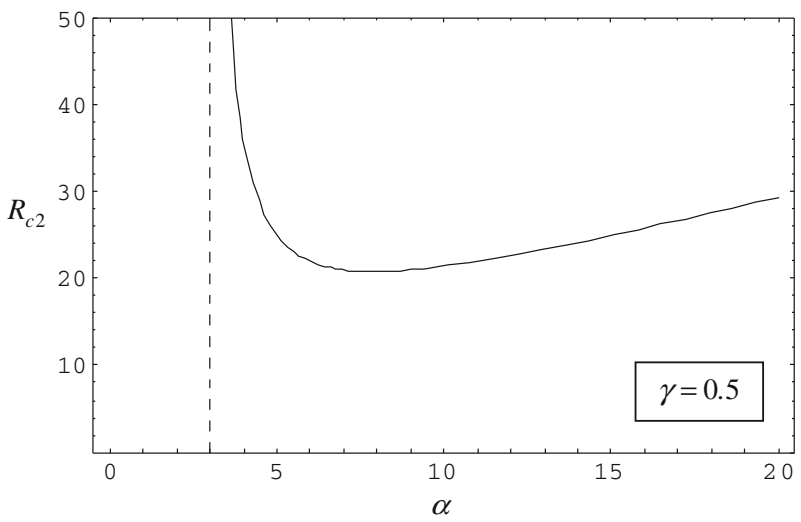

Fig. 2 The dependence of the critical Rayleigh number $R_{c 2}$ on the parameter $\alpha$ is shown for $\gamma=0.5$. The vertical asymptote (dashed line) is at $\alpha=1+4 \gamma=3$, and the coordinates of the absolute minimum of $R_{c 2}$ are $\left(\alpha_{\min }, R_{c 2, \min }\right)=(7.9,20.8)$

\subsection{The Threshold to Turbulence, $R=R_{c 2}$}

Similar to $\bar{R}$, physically it is also interesting to consider the critical Rayleigh number $R_{c 2}$ as a function of the parameter $\alpha$. Owing to the explicit relationship (23), however, this is a substantially easier task. In Fig. 2 the positive branch of $R_{c 2}$ given by the first Eq. 23 is plotted as a function of the (positive) values of $\alpha$ for $\gamma=0.5$.

As already emphasized, $R_{c 2}$ is positive only in the range $\alpha \geq 1+4 \gamma$, and goes to $+\infty$ as $\alpha$ approaches the value $1+4 \gamma$ from above. The dashed line of Fig. 2 shows the corresponding vertical asymptote. In the range $\alpha>1+4 \gamma$, the function $R_{c 2}(\alpha)$ possesses an absolute minimum

$$
R_{c 2, \min }=5+12 \gamma+4 \sqrt{(1+4 \gamma)(1+2 \gamma)}
$$

which is reached at

$$
\alpha \equiv \alpha_{\min }=1+4 \gamma+2 \sqrt{(1+4 \gamma)(1+2 \gamma)}
$$


For $\gamma=0.5$ one has $R_{c 2 \text {, } \min }=11+4 \sqrt{6}=20.8$ and $\alpha_{\min }=3+2 \sqrt{6}=7.9$. For $\alpha=\alpha_{\min }$, the threshold value $\bar{R}$ of the Rayleigh number to spiral nodes is $\bar{R}=1.267125$ and the corresponding eigenvalues are $\lambda_{1}=-8.95762$ and $\lambda_{2}=\lambda_{3} \equiv \bar{\lambda}=-0.970682$. As expected, the value $\bar{R}=1.267125$ is very close to the horizontal asymptote $\bar{R}(\infty)=1.25$ of the green curve shown in Fig. 1. Already this example shows that $\bar{R}$ always is much smaller than $R_{c 2 \text {, min }}$. Indeed, only the green-branch of the curve $\bar{R}(\alpha)$ can cross the domain of existence $\alpha>1+4 \gamma$ of $R_{c 2}(\alpha)$, where this branch lies far below even of $R_{c 2 \text {, min }}(\alpha)$. In the case $\gamma=0.5$, one has $R_{c 2}(\alpha)-\bar{R}>(11+4 \sqrt{6})-1.267125=19.53$. Equation 41 and the first Eq. 32 imply that even for $\alpha \rightarrow \infty$, the inequality $R_{c 2}(\alpha)-\bar{R}>4+11.5 \gamma+4 \sqrt{(1+4 \gamma)(1+2 \gamma)}>8$ holds. In other words, the existence domain $1<\bar{R}<R<R_{c 2}$ of the spiral nodes is quite large for all $\alpha$. It is seen that $R_{c 2}(\alpha)$ is a one-valued function of $\alpha$. Its inverse function, however, is two-valued. This means that every given value $R_{c 2}>R_{c 2 \text {, min }}$ of the critical Rayleigh number is associated with two different conjugate values $\alpha$. In the case $\gamma=0.5$, the conjugate values of $\alpha$ associated with the same value $R_{c 2}=22$, e.g., are $\alpha_{1}=6$ and $\alpha_{2}=11$. The consequences of the existence of a minimum value of $R_{c 2}(\alpha)$ will be discussed in Sect. 4.4 in detail.

\section{Dynamics of the Oscillators}

\subsection{The Potential Energy Picture}

Equation 28 of the overdamped oscillator is a non-homogeneous linear differential equation of the first order. Accordingly, its solution satisfying the pertinent initial condition can easily be obtained in terms of $\xi(t)$ and has the form

$$
\eta(t)=\eta_{0} e^{-4 \gamma t}+2(\alpha-2 \gamma) e^{-4 \gamma t} \int_{0}^{t} \xi^{2}(t) e^{4 \gamma t} \mathrm{~d} t
$$

Equation 7 of the damped Duffing oscillator (coupled to the overdamped one) can be transcribed into the form

$$
\ddot{\xi}=-(1+\alpha) \dot{\xi}-\frac{\partial W}{\partial \xi}
$$

where

$$
W=\frac{1}{4}(R-1)\left[\xi^{4}-2(\alpha-\eta) \xi^{2}\right]
$$

Equation 44 describes the motion of a particle of coordinate $\xi$, mass $m=1$ and potential energy $W$ in the presence of a viscous damping force $-(1+\alpha) \dot{\xi}$, the damping coefficient $(1+\alpha)$ being always positive. The external force which acts on this " $\xi$-particle" is $-\partial W / \partial \xi$. The coordinate $\eta$ of the overdamped oscillator, the " $\eta$-particle", plays in the potential energy $W$ of the $\xi$-particle the role of a parameter. When $R>1$ and $\eta<\alpha, W$ has a double-well shape which is symmetric with respect to $\xi \rightarrow-\xi$ (see Fig. 3). The minima of the two wells are

$$
W_{\min }=-\frac{1}{4}(R-1)(\alpha-\eta)^{2}
$$

and correspond to $\xi= \pm \sqrt{\alpha-\eta}$. The conduction stationary point $\left(\xi_{1}, \eta_{1}\right)=(0,0)$ corresponds to the central maximum $W_{\max }=0$ at $\xi=0$, while the convection stationary points 


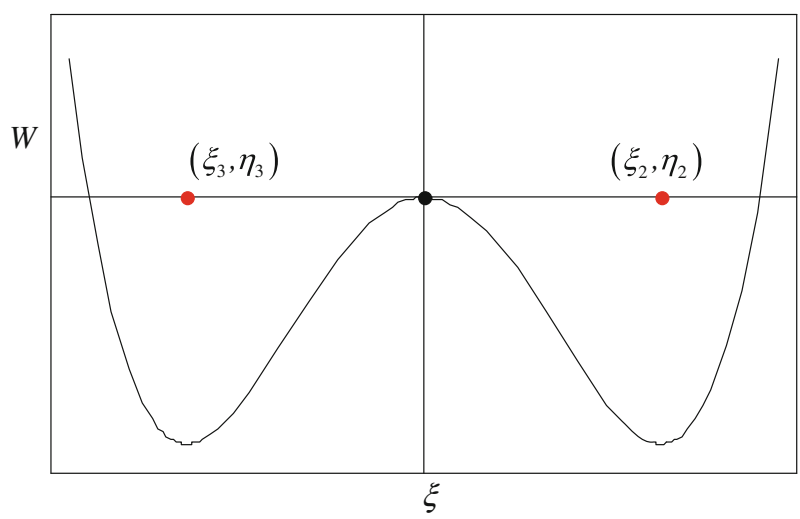

Fig. 3 Plot of the double-well potential energy (45) for $R>1$. The stationary points $\left(\xi_{2,3}, \eta_{2,3}\right)=$ $( \pm \sqrt{2 \gamma}, \alpha-2 \gamma)$ and $\left(\xi_{1}, \eta_{1}\right)=(0,0)$ correspond to the lateral minima $W_{\min }=-(R-1) \gamma^{2}$ and to the central maximum $W_{\max }=0$, respectively

$\left(\xi_{2,3}, \eta_{2,3}\right)=( \pm \sqrt{2 \gamma}, \alpha-2 \gamma)$ correspond to the lateral minima $W_{\min }=-(R-1) \gamma^{2}$ of $W$ at $\xi= \pm \sqrt{2 \gamma}$. In the double-well potential $W$, three families of oscillatory solutions can occur. These are associated with separated oscillations around the minima of the two lateral wells, and some overall oscillations across the central maximum with excursions in the lateral wells, respectively. The trajectories on a chaotic attractor of the Vadasz-Olek model always correspond to the particle motions traveling over these three families of oscillatory states (see also below).

\subsection{The Case $R=1$ of Neutral Stability}

In this case, $W=0$ and Eq. 44 reduces to $\ddot{\xi}+(1+\alpha) \dot{\xi}=0$ which admits the exact solution

$$
\xi=\xi_{0}+\frac{\dot{\xi}_{0}}{1+\alpha}\left(1-e^{-(1+\alpha) t}\right)
$$

Thus, Eq. 43 yields the corresponding $\eta$-solution as

$$
\begin{aligned}
\eta= & \eta_{0} e^{-4 \gamma t} \\
& +2(\alpha-2 \gamma)\left[\left(\xi_{0}+\frac{\dot{\xi}_{0}}{1+\alpha}\right)^{2} \frac{1-e^{-4 \gamma t}}{4 \gamma}-\frac{2 \dot{\xi}_{0}}{1+\alpha}\left(\xi_{0}+\frac{\dot{\xi}_{0}}{1+\alpha}\right)\right. \\
& \left.\times \frac{e^{-(1+\alpha) t}-e^{-4 \gamma t}}{4 \gamma-\alpha-1}+\frac{\dot{\xi}_{0}^{2}}{(1+\alpha)^{2}} \frac{e^{-2(1+\alpha) t}-e^{-4 \gamma t}}{4 \gamma-2 \alpha-2}\right]
\end{aligned}
$$

Equations 47 and 48 describe non-oscillatory motions of the two oscillators from the initial state $\left(\xi_{0}, \dot{\xi}_{0}, \eta_{0}\right)$ toward the uniform asymptotic state

$$
\xi_{\infty}=\xi_{0}+\frac{\dot{\xi}_{0}}{1+\alpha}, \quad \dot{\xi}_{\infty}=0, \quad \eta_{\infty}=\frac{\alpha-2 \gamma}{2 \gamma} \xi_{\infty}^{2}
$$

reached as $t \rightarrow \infty$. As has been shown in Sect. 3.2, in case $R=1$, there exists a continuous infinity of marginally stable stationary points specified by Eq. 12 . The asymptotic state (49) is one of these stationary points. Accordingly, the solution (47), (48) which is pinned on the 
stationary point (49) is also a marginally stable solution of the initial value problem (7), (8), (10).

\subsection{The Subcritical Dynamics in the Range $(\alpha \leq 1+4 \gamma, R>1)$}

As pointed out in Sects. 3.3 and 3.7, the stationary points $\left(\xi_{2,3}, \eta_{2,3}\right)$ are stable for all $R>1$ when $\alpha \leq 1+4 \gamma$. This means that in the parameter range $\alpha \leq 1+4 \gamma$ the oscillation dynamics is always subcritical, i.e., no chaotic oscillations can occur. When $\alpha>1+4 \gamma$, however, the subcritical dynamics is limited to the bounded range $1<R<R_{c 2}$ of the Rayleigh number $R$. The goal of this Section is to examine the subcritical dynamics of the system in the unbounded range $R>1$ where $\alpha \leq 1+4 \gamma$. The subcritical behavior in the bounded range $1<R<R_{c 2}$ with $\alpha>1+4 \gamma$ will be considered in Sect. 4.4.

For the sake of transparency, in all the forthcoming examples for the initial positions of the oscillators the same values

$$
\xi_{0}=\xi_{2}=\sqrt{2 \gamma}, \quad \eta_{0}=\eta_{2,3}=\alpha-2 \gamma
$$

will be adopted. This means that the motion always starts in the point corresponding to the minimum of the right well of the potential energy $W$ (see Fig. 3).

As a first illustration of the subcritical dynamics, we consider the case $\alpha=2 \gamma$ already addressed to in Sect. 3.5. This case is especially transparent since for $\alpha=2 \gamma$, Eq. 43 yields the explicit solution

$$
\eta=\eta_{0} e^{-4 \gamma t} \quad(\alpha=2 \gamma)
$$

which describes the smooth relaxation of the overdamped oscillator from the initial state $\eta_{0}$ to the asymptotic state $\eta_{\infty}=0$. However, owing to the choice (50) of the initial conditions, in the present case, $\eta_{0}=0$, so that the $\eta$-particle remains in rest for all times. Accordingly, Eq. 7 of the $\xi$-particle reduces to the equation of a damped Duffing oscillator of stiffness $-2 \gamma(R-1)$ and damping coefficient $(1+2 \gamma)$,

$$
\ddot{\xi}+(1+2 \gamma) \dot{\xi}+(R-1)\left(-2 \gamma \xi+\xi^{3}\right)=0 \quad(\alpha=2 \gamma)
$$

For $\gamma=1 / 2$, we have $\alpha=1,\left(\xi_{2,3}, \eta_{2,3}\right)=( \pm 1,0),\left(\xi_{0}, \eta_{0}\right)=(1,0)$, and the threshold value of $R$ to the spiral nodes is $\bar{R}=3 / 2$. It is expected in this case that the $\xi$-particle remains within the right well $W$ for all times when its initial velocity $\dot{\xi}_{0}$ is small enough. More precisely, the $\xi$-particle can only move from the right into the left well when its initial kinetic energy $\dot{\xi}_{0}^{2} / 2$ is sufficient to overcome the energy barrier $\left|W_{\min }\right|=(R-1) \gamma^{2}$ between the two minima of $W$ and, at the same time, to supply the energy dissipated by viscous damping, i.e., when

$$
\frac{\dot{\xi}_{0}^{2}}{2}>(R-1) \gamma^{2}+(1+2 \gamma) \int_{0}^{\infty} \dot{\xi}^{2} \mathrm{~d} t \quad(\alpha=2 \gamma)
$$

The equality case of Eq. 53 corresponds to the situation in which the $\xi$-particle comes to rest already at the local maximum of $W$ at $\xi=0$.

In Fig. 4 two solutions of Eq. 52 have been plotted for $\gamma=1 / 2$ and $R=1.2<\bar{R}=1.5$. Both of them start from the same initial position $\xi_{0}=1$, but with different negative initial velocities $\dot{\xi}_{0}$. The red curve has been obtained for $\dot{\xi}_{0}=-2.06382$. This is precisely the situation in which Eq. 53 becomes an equality and thus, for this initial velocity, the $\xi$-particle comes to rest at $\xi=0$. In other words, the red curve coincides with the separatrix which 


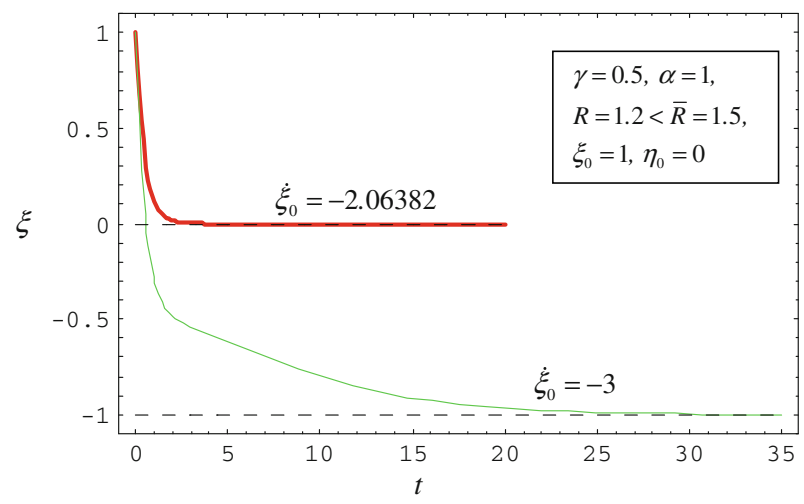

Fig. 4 Two solutions of the Duffing equation (52) corresponding to different initial velocities $\dot{\xi}_{0}$ of the $\xi$ oscillator are shown. The red curve illustrates the situation in which the particle comes to rest at the central maximum of $W$. The green curve corresponds to the case in which the particle passes over the central maximum and approaches the left spiral node $\xi_{3}=-1$ smoothly

separates the families of solutions describing disjointed motions of the particle in the two wells of the potential energy $W$. This separatrix can be approximated by the exponential function

$$
\xi_{\text {sep }}=\xi_{0} \exp \left[-\frac{1}{2}\left(1+2 \gamma+\sqrt{(1-2 \gamma)^{2}+8 \gamma R}\right) t\right]
$$

Thus, $\xi_{\text {sep }}=\exp (-2.095 t)$ and yields $\dot{\xi}_{0}=-2.095$ which deviates from the "exact" numerical result $\dot{\xi}_{0}=-2.06382$ by $1.5 \%$ only.

The green curve of Fig. 4 corresponds to $\dot{\xi}_{0}=-3$. Accordingly, in this case, the particle passes over the central maximum $W$ and approaches the left spiral node $\xi_{3}=-1$ smoothly. The passage time $t_{*}$ and the passage velocity $\dot{\xi}_{*}$ across the central maximum are $t_{*}=0.555312$ and $\dot{\xi}_{*}=-0.974329$, respectively.

In Fig. $5 \mathrm{a}$ and $\mathrm{b}$ the subcritical dynamics in the range $\alpha<1+4 \gamma, R>\bar{R}>1$ is illustrated for the parameter values $\gamma=0.5, \alpha=2.5, R=10$. The initial conditions are $\xi_{0}=1, \dot{\xi}_{0}=8, \eta_{0}=1.5$. The threshold value of $R$ in this case is $\bar{R}=1.31418$, so that the stationary points $\left(\xi_{2,3}, \eta_{2,3}\right)=( \pm 1,1.5)$ are stable spiral nodes. The blue curve of Fig. 5a shows the damped oscillations of the $\eta$-particle around $\eta_{0}=\eta_{2,3}=1.5$. According to the red curve, the $\xi$-particle moves first from its initial position $\xi_{0}=\xi_{2}=1$ to the right, reaches a turning point where its velocity changes sign, then passes the central maximum of $W$, and finally approaches the stable spiral node $\xi_{3}=-1$ by damped oscillations. The passage time $t_{*}$ and the passage velocity $\dot{\xi}_{*}$ across the central maximum are $t_{*}=0.71086$ and $\dot{\xi}_{*}=-1.88403$, respectively. The corresponding position and velocity of the $\eta$-particle are $\eta_{*}=1.61404$. and $\dot{\eta}_{*}=-3.22809$, respectively. The parametric plot $(\xi(t), \eta(t))$ of Fig. $5 \mathrm{~b}$ shows a full agreement with the motions illustrated in Fig. 5a.

\subsection{Along the Shortest Route to Chaos}

In this Section, the motion of the coupled oscillators will be examined in the bounded subcritical range $1<R<R_{c 2}$ with $\alpha>1+4 \gamma$, assuming that $\alpha$ equals the value $\alpha_{\min }=$ $3+2 \sqrt{6}=7.9$ corresponding to the minimum $R_{c 2 \text {, } \min }=11+4 \sqrt{6}=20.8$ of the critical Rayleigh number for $\gamma=1 / 2$ (see Fig. 2). Thus, the variation of $R$ along the vertical line 
(a)

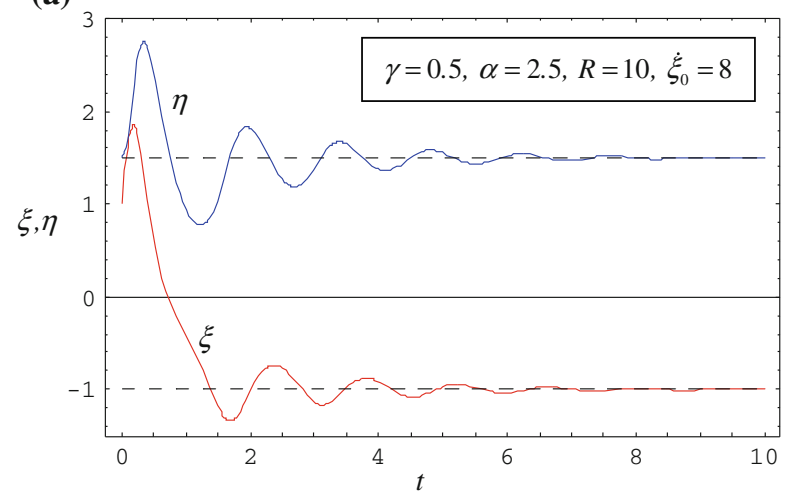

(b)

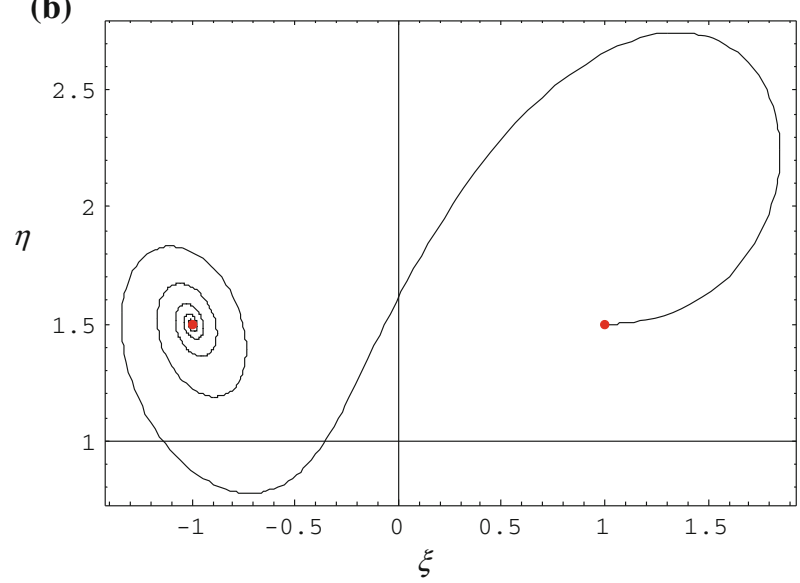

Fig. 5 a The variations with time of the $\xi$ and $\eta$ coordinates for the indicated values of the parameters are shown. In this case, $\xi_{0}=1, \eta_{0}=1.5$, and $R>\bar{R}=1.31418$. b Parametric plot of the coordinates $\xi$ and $\eta$ for the subcritical motion of the coupled oscillators shown in Fig. 5a. The red dots correspond to the stationary points $\left(\xi_{2,3}, \eta_{2,3}\right)=( \pm 1,1.5)$, i.e., to the minima of the double-well potential $W$

$\alpha=7.9$ of the parameter plane $(\alpha, R)$ corresponds to the shortest route toward the chaos, i.e., to the turbulent convection regime of the Vadasz-Olek model for $\gamma=1 / 2$. As mentioned already in Sect. 3.8, in this case, the threshold value $\bar{R}=1.267125$ of the Rayleigh number to spiral nodes is very close to the horizontal asymptote $\bar{R}(\infty)=1.25$ of the green curve of Fig. 1. For this reason, the "interesting" range of $R$ along the shortest route to turbulence is $1.267125<R \leq R_{c 2 \text {, } \min }=20.8$ and this range is quite large.

As illustrations, we consider three stations along the subcritical route $(\alpha=7.9,1.267125<$ $R \leq 20.8$ ) which correspond to $R=10,15$, and $R=R_{c 2, \min }=20.8$, respectively. The initial conditions are the same in all cases, namely $\xi_{0}=\sqrt{2 \gamma}=1, \dot{\xi}_{0}=10, \eta_{0}=\alpha_{\max }-2 \gamma=$ 6.9. The corresponding parametric plots $(\xi(t), \eta(t))$ are shown in Figs. 6, 7, and 8, respectively. For the sake of comparison, in Fig. 9, the parametric plot of the supercritical case $R=22$ is shown under the same conditions. The total duration of motion in all the four cases is $t_{\text {tot }}=20$. It is seen that for $R=10$ and $R=15$ (Figs. 6, 7) where the stationary points $\left(\xi_{2,3}, \eta_{2,3}\right)=( \pm 1,6.9)$ are stable spiral nodes, the $\xi$-oscillator cannot leave the 


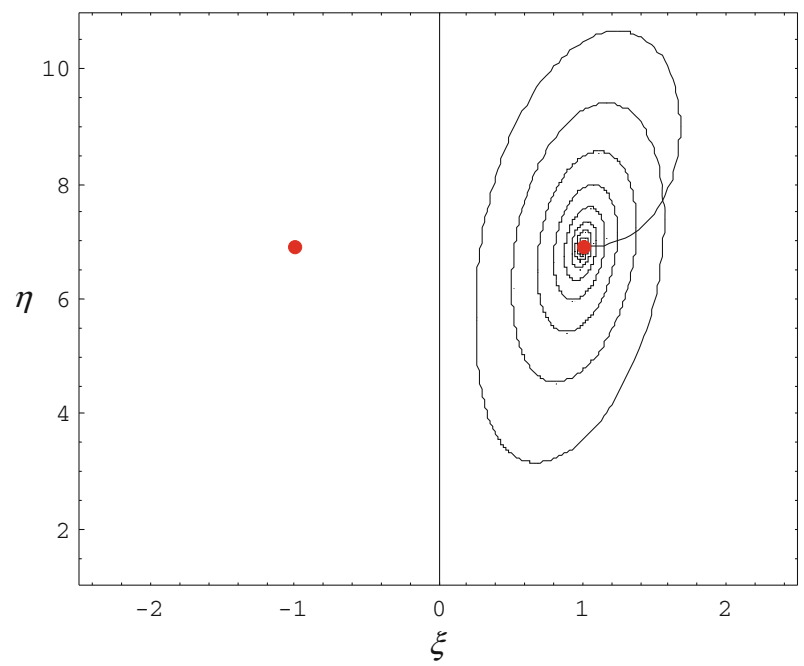

Fig. 6 Parametric plot of the coordinates $\xi$ and $\eta$ for the motion of the coupled oscillators in the subcritical case, $R=10$ (for $\gamma=0.5, \alpha=7.9 \quad \xi_{0}=1, \dot{\xi}_{0}=10, \eta_{0}=6.9, t_{\max }=20$ )

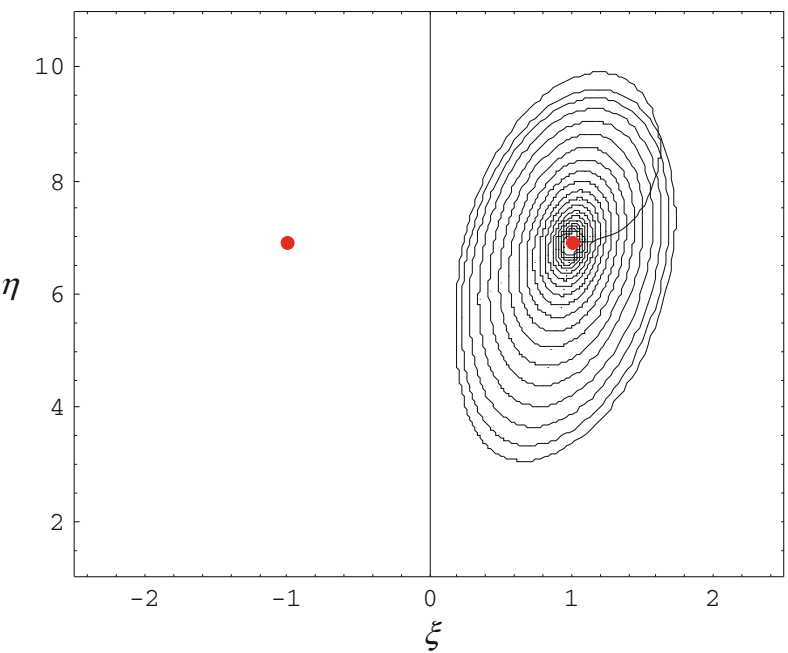

Fig. 7 Parametric plot of the coordinates $\xi$ and $\eta$ for the motion of the coupled oscillators in the subcritical case, $R=15$ (for $\gamma=0.5, \alpha=7.9 \quad \xi_{0}=1, \dot{\xi}_{0}=10, \eta_{0}=6.9, t_{\max }=20$ )

right well of $W$ (where the motion has been started). In the critical and supercritical cases, $R=R_{c 2 \text {, } \min }=20.8$, and $R=22$ of Figs. 8 and 9, however, where the stationary points $\left(\xi_{2,3}, \eta_{2,3}\right)=( \pm 1,6.9)$ are marginally stable and unstable, respectively, overall oscillations across the central maximum with repeated excursions in the lateral wells arise. Moreover, in the supercritical case, $R=22$, the particle spends (until $t=t_{\mathrm{tot}}=20$ ) a longer time around left "eye" of the strange attractor than around the right one, where its motion has been started. 


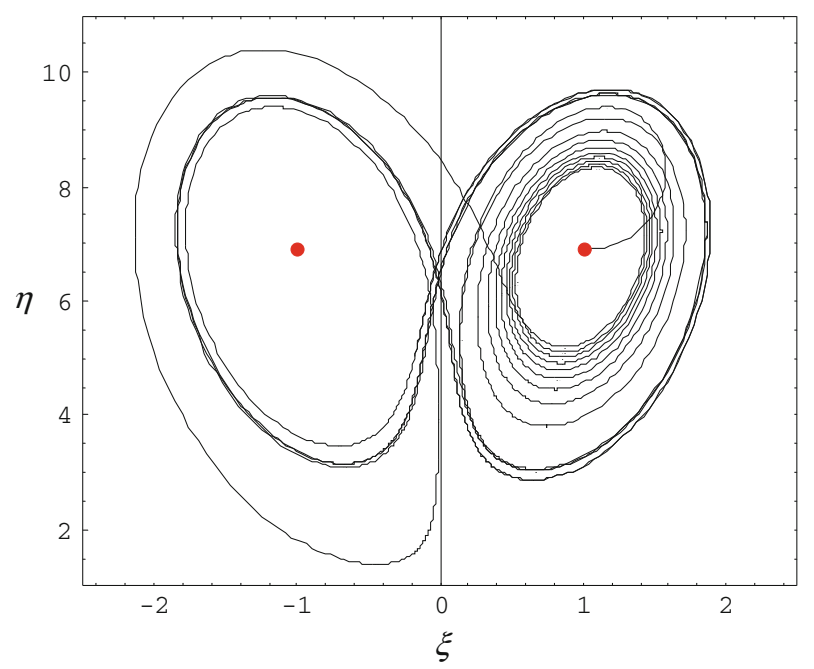

Fig. 8 Parametric plot of the coordinates $\xi$ and $\eta$ for the motion of the coupled oscillators in the critical case, $R=20.8\left(\right.$ for $\left.\gamma=0.5, \alpha=7.9 \quad \xi_{0}=1, \dot{\xi}_{0}=10, \eta_{0}=6.9, t_{\max }=20\right)$

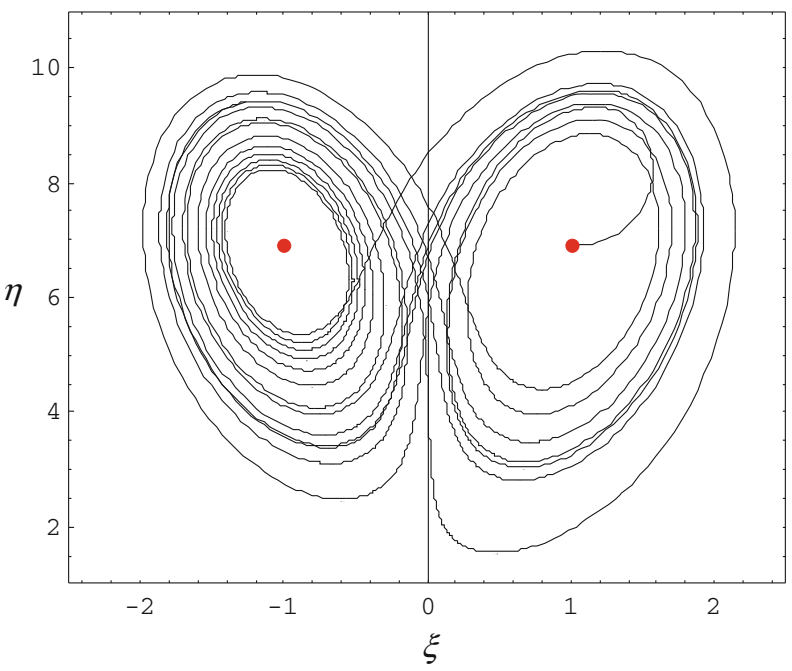

Fig. 9 Parametric plot of the coordinates $\xi$ and $\eta$ for the motion of the coupled oscillators in the supercritical case, $R=22$ (for $\gamma=0.5, \alpha=7.9 \quad \xi_{0}=1, \dot{\xi}_{0}=10, \eta_{0}=6.9, t_{\max }=20$ )

\subsection{The Butterfly Effect}

One of the basic characteristics of the chaotic dynamics is its high sensitivity with respect to the initial conditions. The reason is that in the chaotic regime of a dynamical system, the distance between two initially neighboring trajectories increases exponentially with time. This phenomenon is known under the metaphoric name Butterfly Effect, indicating that in a chaotic dynamical state of the atmosphere, a tiny flap of a butterfly is sufficient to make the evolution unpredictable, and thus the weather forecast becomes impossible (see also Magyari 2010). In the present oscillator-description of the Vadasz -Olek model, the 


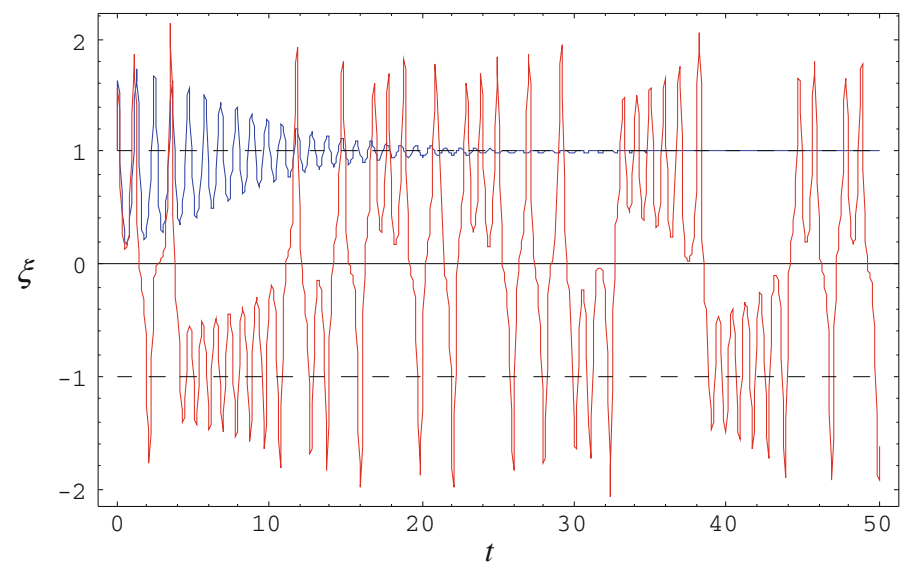

Fig. 10 Damped oscillations of the $\xi$-particle in the subcritical case of Fig. 7 (blue curve) and its chaotic oscillations in the supercritical case of Fig. 9 (red curve)

Butterfly Effect is illustrated in Fig. 11. The red curve of Fig. 11 is identical to the red curve of Fig. 10 which describes the supercritical (chaotic) oscillations of the $\xi$-particle for $\gamma=0.5, \alpha=\alpha_{\max }=7.9 \quad \xi_{0}=1, \dot{\xi}_{0}=10, \eta_{0}=6.9$ and $R=22>R_{c 2 \text {, } \min }=20.8$. The green curve also describes the supercritical oscillations of the $\xi$-particle for the same values of the pertinent parameters, except for the initial velocity which in this case is $\dot{\xi}_{0}=10.01$, and thus deviates from the initial velocity of the former motion (red curve) by $1 \%$ only. It is seen that the two curves are nearly overlapping approximately until $t=2.5$, but for large times they become fully uncorrelated, the oscillations being often localized in different wells of the potential energy $W$.

\subsection{Conjugate Critical Attractors}

As emphasized by Fig. 2, every value $R_{c 2}>R_{c 2 \text {, min }}$ of the critical Rayleigh number is associated with two different of the parameter $\alpha$. These "conjugate values" of $\alpha$ become coincident when $R_{c 2}$ approaches the absolute minimum (41) of the function $R_{c 2}(\alpha)$. In the case of $\gamma=0.5$, the conjugate values of $\alpha$ associated with the same value $R_{c 2}=22$, e.g., are $\alpha_{1}=6$ and $\alpha_{2}=11$. The critical attractors corresponding to these conjugate critical states of the oscillators are plotted in Fig. 12. The red and blue trajectories correspond to $\alpha_{1}=6$ and $\alpha_{2}=11$, respectively. The particles are started in both cases with the same initial velocity $\dot{\xi}_{0}=10$ from the right minimum of $W$, i.e., from the stationary point $(\xi, \eta)=(\sqrt{2 \gamma}, \alpha-2 \gamma)$ which corresponds to the conjugate initial conditions $\left(\xi_{0}, \eta_{0}\right)=(1,5)$ and $\left(\xi_{0}, \eta_{0}\right)=(1,10)$, respectively. Figure 12 shows that, as a consequence of the change from $\alpha_{1}=6$ (red trajectory) to its large conjugate counterpart $\alpha_{2}=11$ (blue trajectory), the critical attractor also undergoes manifest changes. Thus, the "eyes" of the attractor are shifted upward from $\eta_{0,1}=\alpha_{1}-2 \gamma=5$ to $\eta_{0,2}=5 \alpha_{2}-2 \gamma=10$. At the same time, the size of the attractor increases in the $\xi$-direction slightly and $\eta$-direction massively.

\section{Summary and Conclusions}

The Vadasz-Olek model of chaotic convection in a porous layer has been investigated in this article by mapping its basic equations on the governing equations of a damped non- 


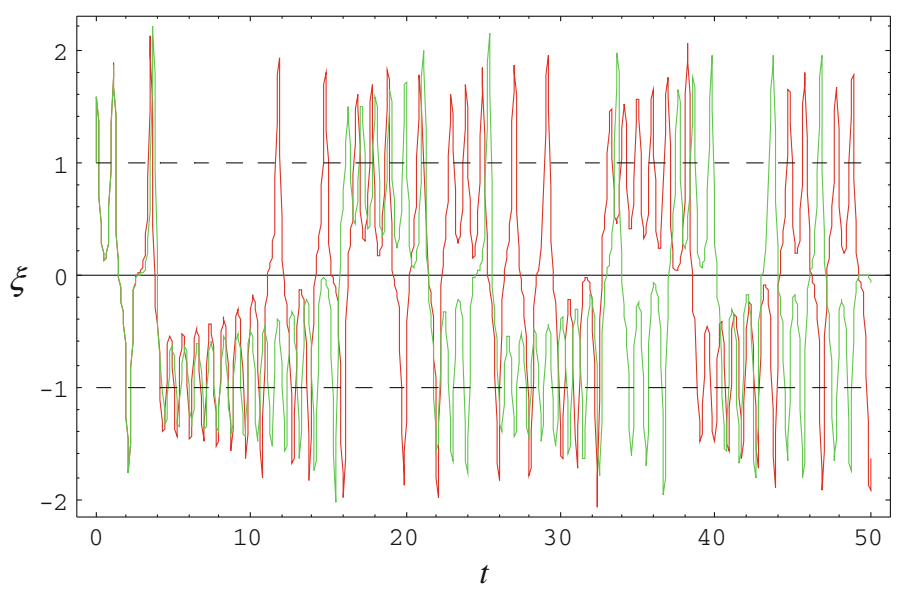

Fig. 11 The Butterfly effect in the Vadasz-Olek model. The two chaotic oscillations correspond to the same values of the pertinent parameters, except for their initial velocities $\dot{\xi}_{0}$ which differ by $1 \%$ from each other

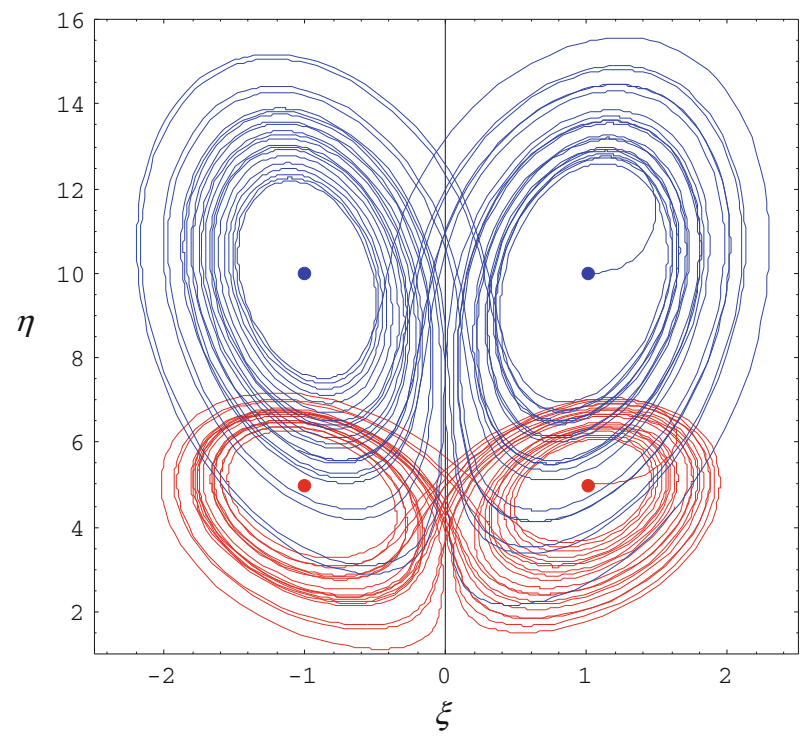

Fig. 12 Critical attractors corresponding to the conjugate values $\alpha_{1}=6$ (red trajectory) and $\alpha_{2}=11$ (blue trajectory) of the parameter $\alpha$ which are associated (for $\gamma=0.5$ ) with the same value of the critical Rayleigh number, $R_{c 2}=22$

linear oscillator, the $\xi$-particle, modulated by a linear degenerated overdamped oscillator (relaxator), the $\eta$-particle. The relaxator is coupled self-consistently to the damped $\xi$-oscillator by a nonlinear cross force. This point-mechanical analogy provides an intuitive insight into the details of the regular and chaotic dynamics. Concerning the convection range $R>1$ of the model, the main results of the article can be summarized as follows:

1. The potential energy $W$ of the $\xi$-particle has a double-well shape (see Eq. 45 and Fig. 3) so that the conduction stationary point $\left(\xi_{1}, \eta_{1}\right)=(0,0)$ corresponds to the central maximum $W_{\max }=0$ at $\xi=0$, while the convection stationary points $\left(\xi_{2,3}, \eta_{2,3}\right)=$ 
$( \pm \sqrt{2 \gamma}, \alpha-2 \gamma)$ correspond to the lateral minima $W_{\min }=-(R-1) \gamma^{2}$ of $W$ at $\xi=$ $\pm \sqrt{2 \gamma}$.

2. In the double-well potential $W$, three families of oscillatory solutions can occur which are associated with separated oscillations around the minima of the two lateral wells, and some overall oscillations across the central maximum with repeated excursions in the lateral wells, respectively. The trajectories on the chaotic attractors of the Vadasz-Olek model always correspond to particle motions traveling over these three families of oscillatory states. The coordinates $( \pm \sqrt{2 \gamma}, \alpha-2 \gamma)$ of the "eyes" of these strange attractors correspond to the lateral minima of the potential energy $W$ of the modulated nonlinear oscillator.

3. As long as $\alpha \leq 1+4 \gamma$, the dynamics of the oscillators is always subcritical., i.e., the convection stationary points $\left(\xi_{2,3}, \eta_{2,3}\right)=( \pm \sqrt{2 \gamma}, \alpha-2 \gamma)$ are stable simple nodes or stable spiral nodes for all $\gamma>0$ and $R>1$.

4. The threshold value $\bar{R}(\alpha ; \gamma)$ of the Rayleigh number $R$ where the convection stationary points change from the simple node to the spiral node behavior, reaches its maximum value when all three negative eigenvalues $\lambda_{i}$ become coincident. For $\gamma=0.5$, this happens at $\alpha=0.852311$ where $\bar{R}_{\max }=1.62107$ (point $B$ of Fig. 1).

5. With increasing values of $\alpha, \bar{R}(\alpha ; \gamma)$ decreases from its maximum value monotonically and approaches the asymptotic value $\bar{R}=1+\gamma / 2$ when $\alpha \rightarrow \infty$ (see Eq. 32 and Fig. 1).

6. Critical $\left(R=R_{c 2}\right)$ and supercritical $\left(R>R_{c 2}\right)$ oscillations are possible only in the range $\alpha \geq 1+4 \gamma$ where the function $R_{c 2}(\alpha)$ possesses an absolute minimum $R_{c 2 \text {, min }}$ reached at a certain $\alpha \equiv \alpha_{\min }$ (see Eqs. 41, 42 and Fig. 2).

7. At the special value $\alpha_{\min }$ of the parameter $\alpha$ and with increasing values of $R$, one encounters the shortest route to chaos, i.e., the shortest route to turbulent flow according to the Vadasz-Olek model. Along this shortest route, the change of the oscillation dynamics, as well as of the corresponding trajectories in the coordinate plane $(\xi, \eta)$, are illustrated in Figs. 6, 7, 8, 9, and 10.

8. In the present oscillator analogy, the Butterfly Effect, i.e., the sensitive dependence of the chaotic dynamics on the initial conditions, becomes clearly manifest (see Fig. 11).

9. The property 6 also implies that every given value $R_{c 2}>R_{c 2}$, min of the critical Rayleigh number is associated with two different conjugate values $\alpha$. (see Fig. 2). The critical attractor undergoes significant changes by passing from one of the conjugate $\alpha$-values to the other (see Fig. 12).

In addition to its physical transparency, the mathematical advantage of the point-mechanical analogy reported in this article consists of the applicability of several analytical approximation methods known from the theory of nonlinear oscillations. This offers a further research opportunity for the investigation in the future on the Vadasz-Olek model.

\section{References}

Dauxois, T., Fermi, P.: Ulam and a mysterious lady. Phys. Today 61, 55-57 (2008)

Eckmann, J.P.: Roads to turbulence in dissipative dynamical systems. Rev. Mod. Phys. 53, 643-654 (1981)

Feigenbaum, M.J.: The universal metric properties of nonlinear transformations. J. Stat. Phys. 21, 669706 (1979)

Haken, H.: Analogy between higher instabilities in fluids and lasers. Phys. Lett. A 53, 77-78 (1975)

Holmes, P.: A nonlinear oscillator with a strange attractor. Philos. Trans. R. Soc. Lond. A 292, 419-448 (1979)

Kneubühl, F.K.: Oscillations and Waves. Springer, Berlin (1997)

Li, T.-Y., Yorke, J.A.: Period three implies chaos. Am. Math. Monthly 82, 985-992 (1975)

Lorenz, E.N.: Deterministic nonperiodic flow. J. Atmos. Sci. 20, 130-141 (1963) 
Magyari, E.: The "Butterfly Effect" in a porous slab. Transp. Porous Med.(2010). doi:10.1007/ s11242-010-9536-5

May, R.M.: Simple mathematical models with very complicated dynamics. Nature 261, 459-467 (1976)

Rössler, O.E.: An equation for continuous chaos. Phys. Lett. A 57, 397-398 (1976)

Rössler, O.E.: An equation for hyperchaos. Phys. Lett. A 71, 155-157 (1979)

Schuster, H.G.: Deterministic Chaos. Physik-Verlag, Weinheim (1984)

Shimizu, T., Morioka, N.: Chaos and limit cycles in the Lorenz model. Phys. Lett. A 66, 182-184 (1978)

Stoop, R., Steeb, W.H.: Berechenbares Chaos in dynamischen Systemen. Birkhäuser Verlag, Basel (2006)

Vadasz, P.: Local and global transitions to chaos and hysteresis in a porous layer heated from below. Transp. Porous Media. 37, 213-245 (1999)

Vadasz, P.: Small and moderate Prandtl number convection in a porous layer heated from below. Int. J. Energy Res. 27, 941-960 (2003)

Vadasz, P: Analytical prediction of the transition to chaos in Lorenz equations. Appl. Math. Lett. 23, 503-507 (2010). doi:10.1016/j.aml.2009.12.012

Vadasz, P., Olek, S.: Transitions and chaos for free convection in a rotating porous layer. Int. J. Heat Mass Transf. 41, 1417-1435 (1998)

Vadasz, P., Olek, S.: Weak turbulence and chaos for low Prandtl number gravity driven convection in porous media. Transp. Porous Media. 37, 69-91 (1999)

Vadasz, P., Olek, S.: Route to chaos for moderate Prandtl number convection in a porous layer heated from below. Transp. Porous Media. 41, 211-239 (2000) 